\title{
Transmission Electron Microscopy Studies of Electrical Active GaAs/GaN Interface Obtained by Wafer Bonding
}

\author{
J. Jasinski, Z. Liliental-Weber, S. Estrada ${ }^{1}$, E.Hu ${ }^{1}$ \\ Materials Science Division, Lawrence Berkeley National Laboratory, Berkeley, CA 94720, USA \\ ${ }^{1}$ Materials Department, University of California, Santa Barbara, CA 93106
}

\begin{abstract}
Transmission electron microscopy (TEM) and energy dispersive X-ray spectroscopy (EDX) studies of GaAs/GaN interfaces, obtained by direct wafer bonding, are presented. TEM observations show that most of the interface area was well bonded. A thin oxide layer, confirmed by EDX, was present at the interface in the well-bonded regions. Plan-view TEM studies showed the presence of two dislocation networks in such regions. They formed to accommodate: (1) tilt between bonded crystals and (2) strain, which appeared during sample cooling due to mismatch in thermal expansion coefficients. Asymmetrical, often elongated, cavities, formed on the GaAs side, were present at the interface between the well-bonded regions. It was shown by EDX that the walls of these cavities are covered with native oxide.
\end{abstract}

\section{INTRODUCTION}

Wafer bonding is a well-recognized method for semiconductor integration. It has been successfully applied to $\mathrm{Si}$ on $\mathrm{Si}$ integration [1] and to a number of lattice mismatched material systems such as: $\mathrm{GaAs} / \mathrm{Si}$ [2], InP/GaAs [3-5], InP/GaN [6] and GaAs/sapphire [7,8]. Rapid development in research and application of $\mathrm{GaN}$ and related materials has resulted in increased interest in the possible integration of $\mathrm{GaN}$ with other semiconductors, among which GaAs plays an important role. Successful wafer bonding of these two materials would allow integration of GaAs and $\mathrm{GaN}$ based optoelectronic devices. A GaAs/GaN interface combines also the high breakdown voltage of GaN with the high mobility of GaAs, which are ideal characteristics for transistors and other electronic devices. Promising attempts to apply direct wafer bonding, to create an electrically active $\mathrm{GaAs} / \mathrm{GaN}$ interface have recently been reported $[9,10]$. In this paper we present transmission electron microscopy studies of these same interfaces.

\section{EXPERIMENTAL RESULTS}

GaN layers with a nominal thickness of $2 \mu \mathrm{m}$ were grown on c-plane (0001) sapphire substrates by metal organic chemical vapor deposition (MOCVD). They were directly bonded with $1 \mu \mathrm{m}$-thick GaAs layers grown by molecular beam epitaxy (MBE) on $0.3 \mu \mathrm{m}$-thick MBE AlAs etch-stop layers grown on (001) GaAs substrates. Atomic force microscopy revealed the rms roughness of both GaAs and GaN surfaces to be about $0.4 \mathrm{~nm}$. Prior to bonding, "escape channels" were etched into GaAs, to prevent liquid and gas from being trapped at the interface when $\mathrm{GaAs}$ and $\mathrm{GaN}$ were later brought together. These channels were $10 \mu \mathrm{m}$ wide, in a rectangular grid of $150 \mu \mathrm{m} \times 400 \mu \mathrm{m}$. Before bonding the wafers were cleaved into $1 \mathrm{~cm} \times 1 \mathrm{~cm}$ pieces, cleaned, joint together in solvent and kept at $750^{\circ} \mathrm{C}$ for up to 1 hour in a nitrogen ambient under uniaxial pressure of $2 \mathrm{MPa}$. In the next step, when the pressure was released and the bonded structure was again at room temperature the entire GaAs substrate and the AlAs layer 
were removed by wet etching. This resulted in the following layer structure: GaAs $(1 \mu \mathrm{m}) / \mathrm{bonded}$ interface/GaN $(2 \mu \mathrm{m}) /$ sapphire. Recent Secondary Ion Mass Spectroscopy (SIMS) study of these bonded $\mathrm{GaAs} / \mathrm{GaN}$ interfaces revealed high $\mathrm{Si}, \mathrm{Be}, \mathrm{O}$ and $\mathrm{C}$ signals, which broaden with increasing bonding time or temperature [11].

Cross-sectional as well as plan-view TEM specimens were prepared from fused samples using the standard method of mechanical pre-thinning followed by Ar-ion milling. An OO2B TOPCON microscope operated at $200 \mathrm{kV}$ was used to study the microstructure of the fused interfaces. The chemical composition of these interfaces was investigated by energy dispersive X-ray spectroscopy (EDX). A CM200 Philips microscope with a field emission gun, operated at $200 \mathrm{keV}$ and with a beam size of $1.2 \mathrm{~nm}$ was used for this study.

\section{Well-bonded regions}

Both, cross-sectional and plan-view studies revealed that most of the fused interface area was well-bonded and the quality of the GaAs/GaN interface in such regions was very high. High resolution electron microscopy (HREM) images recorded in such well-bonded regions showed a uniform interface between the two bonded wafers (see Fig. 1(a)). The only imperfection observed at this interface was a thin, bright layer with a thickness in the range of 5-10 $\AA$. This suggests that a thin amorphous layer was present at this interface.

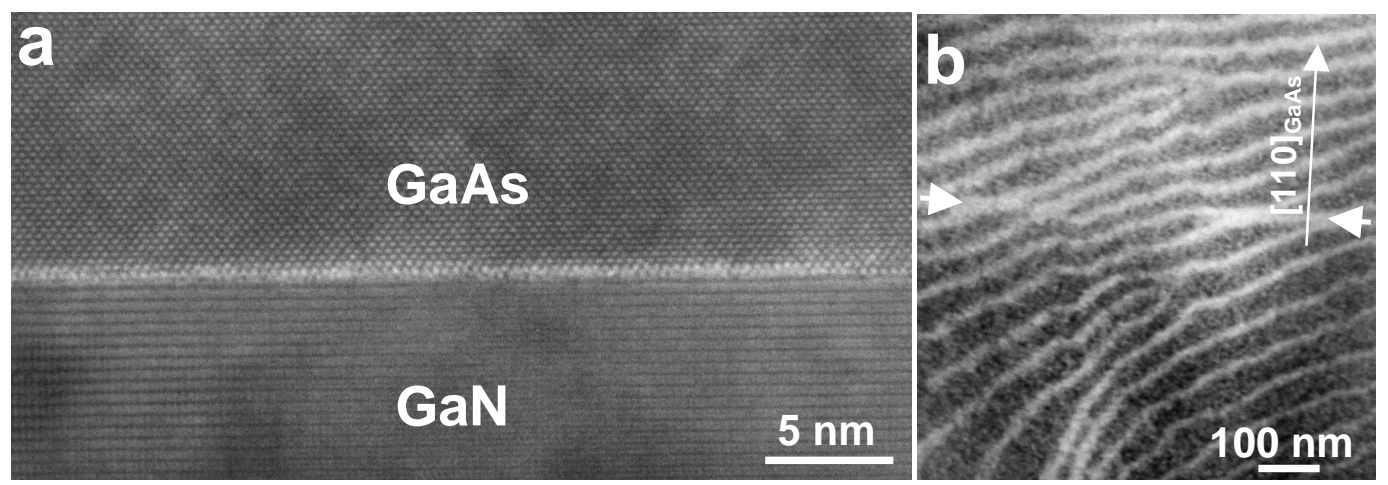

Fig. 1. (a) HREM image of the GaAs/GaN interface in a well-bonded region. (b) Plan-view TEM image of such a well-bonded region.

In order to determine the chemistry of this layer EDX spectra were obtained at the GaAs/GaN bonded interface. One such spectrum is shown in Fig. 2(a). In addition to X-ray peaks coming from $\mathrm{Ga}$, As and $\mathrm{N}$ we observed $\mathrm{O}$ and $\mathrm{C}$ peaks with significant intensity. The presence of carbon is most probably due to specimen contamination inside the microscope. We found that the intensity of this peak was similar for different places on the specimen. In the case of oxygen the situation was different. High intensity of the oxygen peak was detected only at the bonded interface. This can be seen in Fig. 2(b). The line profiles of $\mathrm{N}$ and As peaks as well as a line profile of background intensity are included in this figure for reference. Intensity line profiles of the As and $\mathrm{N}$ peaks clearly show the location of the interface between GaAs and GaN layers. The line profile of the background intensity contains information about relative changes of specimen thickness. Since the background intensity has almost a constant value we can conclude that the specimen had a constant thickness in the area of the measurement. From analysis of the $\mathrm{O}$ line profile shown in Fig. 2(b) one can see that the intensity of the $\mathrm{O}$ peak 
follows background intensity in areas away from the interface - on either the GaAs or the GaN sides. On the other hand, there is a significant increase of the $\mathrm{O}$ peak intensity exactly at the interface position. This result is consistent with the HREM observations and indicates that a thin native oxide layer is present at the GaAs/GaN bonded interface. The thickness of the oxidized layer, as read directly form EDX profiles was about $10 \mathrm{~nm}$. However, it was overestimated because of the TEM foil thickness and the associated beam spread. The influence of this effect on the broadening of interfaces can be seen in the broadening of the threshold in the As and $\mathrm{N}$ profiles (see Fig. 2(b)). The width of these thresholds, as read directly form EDX, was also about $10 \mathrm{~nm}$, e.g. about ten times larger that the interface roughness obtained from the HREM study. This indicates that the real thickness of the oxidized layer is much lower - probably in the range of about 1-2 $\mathrm{nm}$.

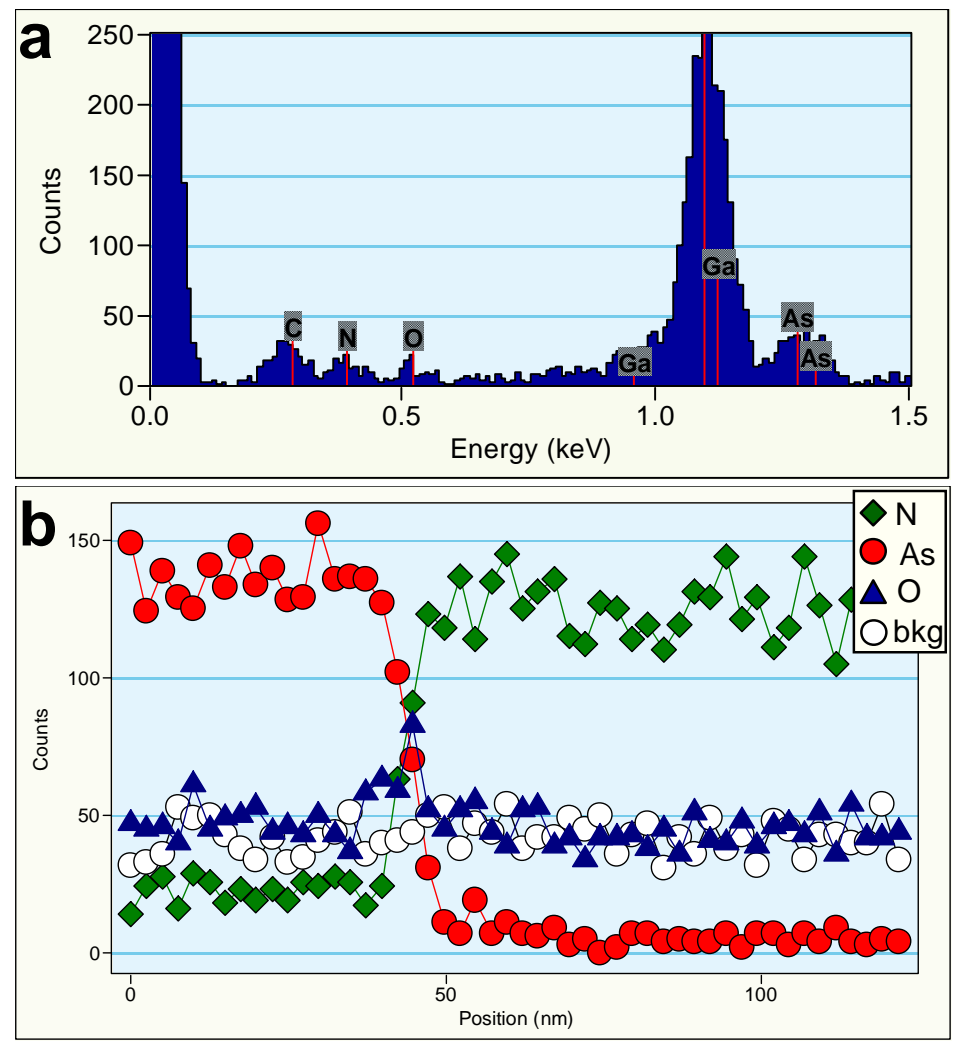

Fig. 2. (a) EDX spectrum recorded at the GaAs/GaN interface in a well-bonded region. (b) EDX line profiles measured across this interface.

Plan-view studies of well-bonded regions of GaAs/GaN interfaces revealed two important features. First is a network of wavy lines, clearly visible in the plan-view image shown in Fig. 1(b). These lines are dislocations formed at steps to accommodate tilt between the two crystals. Since the tilt has two components: an average tilt originating from the miscut between the two crystals and local tilts due to surface roughness these dislocations are primarily oriented along an average direction but locally they often change direction. In addition, since the axis of an unintentional miscut, between two bonded crystals, is accidental the average orientation of this dislocation network changes from sample to sample. The orientation of these dislocations is governed by the direction of the tilt axis, and the separation between them depends on the tilt angle. Again since the magnitude of an unintentional miscut is accidental the spacing between dislocations changes from sample to sample. In addition, surface roughness and local 
irregularities introduce additional local tilts and cause fluctuations of this spacing. The average dislocation spacing, measured for the GaAs/GaN samples was about $50 \mathrm{~nm}$. Similar values have been reported for such dislocation networks at InP/GaAs bonded interfaces [3].

The second feature observed in plan-view images of well-bonded areas was straight lines running along the $[110]_{\mathrm{GaAs}}$ or $[110]_{\mathrm{GaAs}}$ direction. One such line is shown in the Fig. 1(b). These lines, also dislocations, formed a two-dimensional irregularly-spaced network (with an average separation of the order of a few micrometers). They were formed to relieve an additional strain, which resulted from the mismatch in thermal expansion coefficients, when the sample was cooled down from the bonding temperature to room temperature. For GaAs/GaN bonding these dislocations are formed on the GaAs side since they are parallel or perpendicular to the [110] $]_{\mathrm{GaAs}}$ direction. Similar dislocation networks, resulting from the difference in thermal expansion coefficients $\left(\sim 4.5 \times 10^{-6} \mathrm{~K}^{-1}\right.$ for $\operatorname{InP}[3]$ and $\sim 6.5 \times 10^{-6} \mathrm{~K}^{-1}$ for GaAs [12]), have been reported for the $\mathrm{InP} / \mathrm{GaAs}$ system [3,4]. In our study the situation is more complicated since there are three components with different thermal expansion coefficients: sapphire substrate $\left(\sim 8 \times 10^{-6}\right.$

$\left.\mathrm{K}^{-1}[12]\right)$, GaN layer ( $5.6 \times 10^{-6} \mathrm{~K}^{-1}$ for $\mathrm{GaN}$ [13]) and GaAs layer. Nevertheless, the differences between thermal expansion coefficients of these components are of the same order of magnitude as in the case of the InP/GaAs system and similar densities of dislocations, as shown by plan-view TEM studies, are formed in these two cases.

\section{Cavities}

Numerous cavities were found between the well-bonded regions. Almost all of them, except the very small ones, were elongated along the $[110]_{\text {GaAs }}$ direction. Four such cavities are visible on the plan-view TEM image shown in Fig. 3(a). The in-plane size of such cavities varied within a very wide range, covering three orders of magnitude, from a few tens of nanometers to a few tens of micrometers. Cross-sectional studies showed that these cavities were very asymmetric and were entirely formed on the GaAs side. This can be seen on the HREM crosssectional image shown in Fig. 3(b). Height of these cavities varied in the range 5 to $20 \mathrm{~nm}$. HREM study showed that many of these cavities had facets on (001) and $\{111\}$ GaAs planes (see Fig. 3(b)).

EDX line profiles were measured across such cavities in order determine if they are filled and what is their chemistry. In addition to $\mathrm{Ga}$, As and $\mathrm{N}$ peaks, $\mathrm{C}$ and $\mathrm{O}$ peaks were again found in the EDX spectra. As in Fig. 2(b) the line profiles of the O, N and As peaks as well as background intensity, measured across the cavity, are shown in Fig. 3(c). From the shape of the $\mathrm{N}$ peak line profile the location of the GaN surface (e.g. the bottom surface of the cavity) can be determined. It corresponds to the position about $70 \mathrm{~nm}$ on the horizontal scale. One can notice that at this position the $\mathrm{O}$ peak line profile has an intensity peak. A similar peak is also visible at about $35 \mathrm{~nm}$, which corresponds to the location of the top cavity surface. One can also see that within the range of 35 to $70 \mathrm{~nm}$, which corresponds to cavity location, the $\mathrm{O}$ peak intensity is significantly higher than that of the background. On the other hand, outside this range the $\mathrm{O}$ peak intensity almost follows the background intensity. The background intensity, corresponding to TEM specimen thickness, has a significant dip at the cavity location, which suggests that the cavity is at least partially empty. This explains why the As peak intensity has a lower value in the area of the cavity. In summary, all these EDX results indicate that cavities are partially empty, with an oxide layer coating their inner walls. 
Such empty or partially filed asymmetrical cavities, formed at the interface of directly bonded materials, have been reported in the literature for the InP/GaAs [3,4] and GaAs/sapphire $[7,8]$ systems. It seems that they are formed as a result of migration of small voids, which appear at the interface due to wafer surface irregularities, which existed prior to bonding. At elevated bonding temperature these voids can migrate at the interface and agglomerate to reduce the overall non-bonded interface area. The cavity asymmetry is a manifestation of much faster diffusion in GaAs than in GaN. The observed faceting of cavity walls reduces the surface free energy of the system. The oxide layer, detected by the EDX, located on the cavity walls, can originate from a residual native oxide, which existed on the wafer surfaces prior bonding.

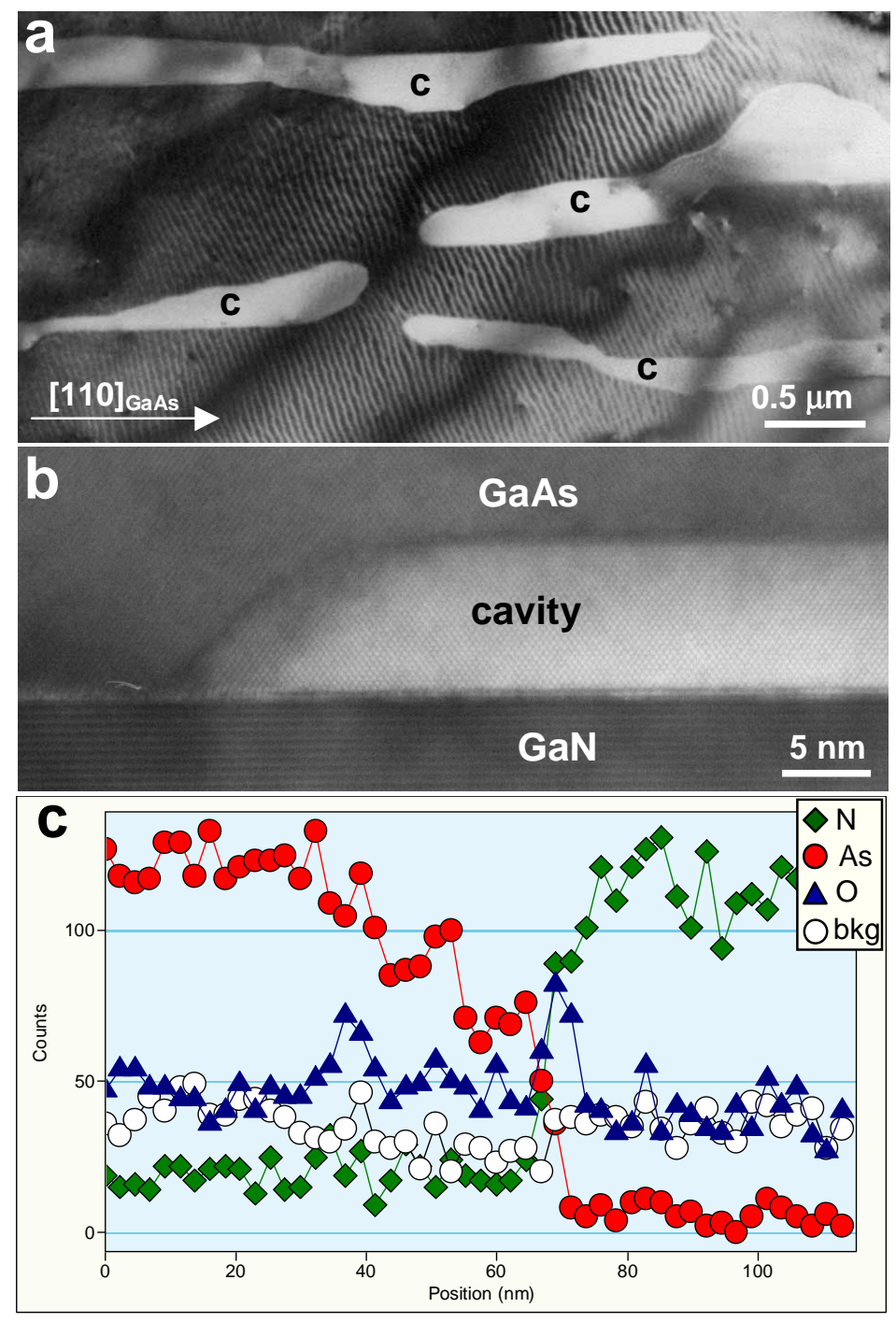

Fig. 3. (a) Plan-view TEM image of the GaAs/GaN bonded sample. Notice four elongated cavities between well-bonded regions. (b) Cross-sectional image of cavity formed at the GaAs/GaN interface. (c) EDX line profiles across the cavity.

\section{CONCLUSIONS}

TEM characterization of $\mathrm{GaAs} / \mathrm{GaN}$ interfaces, obtained by direct wafer fusion, was performed. It was shown that most of the interface area was well bonded with only a thin layer 
(of thickness about 1-2 nm) of residual oxide. The Presence of oxygen at the interface was confirmed by EDX study. Two networks of dislocations were observed in well-bonded regions: one to accommodate a tilt between the two bonded crystals and a second, to accommodate strain, which resulted from sample cooling. Between the well-bonded regions of the interface numerous asymmetrical cavities, formed on the GaAs side, were also observed. Most of them were elongated in the [110] $]_{\mathrm{GaAs}}$ direction and had faceting on the (001) and $\{111\}$ GaAs planes. EDX study showed that walls of such cavities are covered by an oxide layer.

\section{ACKNOWLEDGEMENTS}

This research at LBNL was supported by the Air Force Office of Scientific Research, through the U.S. Department of Energy, Order No. AFOSR-ISSA-99-0012 under the sponsorship of Dr. G. Witt and at UCSB by the DoD Multidisciplinary University Research Initiative (MURI) program administered by the Office of Naval Research under Grant N0001498-1-0654. The authors would like to thank A. Huntington and L. Coldren and, A. Stonas and S. DenBaars from UCSB for the growth of GaAs and GaN, respectively. The authors would like also to thank W. Swider for TEM sample preparation and the National Center for Electron Microscopy at LBNL for the opportunity to use its facilities.

\section{REFERENCES}

1. M. Benamara, A. Rocher, A. Laporte, G. Sarrabayrousse, L. Lescouzeres, A. PeyreLavigne, M. Fnaiech, and A. Claverie, Mater. Res. Soc. Symp. Proc. 378, 863 (1995).

2. Y. C. Zhou, Z. H. Zhu, D. Crouse, and Y. H. Lo, Appl. Phys. Lett. 3, 2337 (1998).

3. G. Patriarche, F. Jeannes, J.-L. Oudar, F. Glas, J. Appl. Phys. 82, 4892 (1997).

4. L. Sagalowicz, A. Rudra, E. Kapon, M. Hammar, F. Salomonsson, A. Black, P.-H. Jouneau, T. Wipijewski, J. Appl. Phys. 87, 4135 (2000).

5. N. Y. Jin-Phillipp, W. Sigle, A. Black, D. Babic, J.E. Bowers, E.L. Hu, M. Ruhle, J. Appl. Phys. 89, 1017 (2001).

6. R. K. Sink, S. Keller, B. P. Keller, D. I. Babic, A. L. Holmes, D. Kapolnek, S. P. DenBaars, and J. E. Bowers, X. H. Wu and J. S. Speck, Appl. Phys. Lett. 68, 2147 (1996).

7. P. Kopperschmidt, G. Kaestner, S. Senz, D. Hesse, U. Goesele, Appl. Phys. A 64, 533 (1997).

8. St. Senz, P. Kopperschmidt, G. Kastner, D. Hesse, J. Mater. Sci. 33, 2073 (1998).

9. S. E. Monteith, L. S. Mc Carthy, S. K. Mathis-Yu, H. Marchand, U. K. Mishra, J. S. Speck, S. P. Den Baars, and E. L. Hu, ""Wafer Fusion of GaAs/GaN Heterostructures", presented at the 42nd Electronic Materials Conference, Denver, CO, 2000 (unpublished).

10. S. E. Monteith, J. Jasinski, A. Huntington, A. Stonas, L. Coldren, S. Den Baars, Z. LilientalWeber, U. Mishra, and E. Hu, "GaAs/GaN Diodes Wafer-Fused at $500^{\circ} \mathrm{C}$ ", presented at the 43rd Electronic Materials Conference, Notre Dame, IN, 2001 (unpublished).

11. S.M. Monteith, L. Zhang, T. Margalith, L. Coldren, S. DenBaars, U. Mishra, E. Hu (to be published).

12. Y.S. Touloukian, R.K. Kirby, R.E. Taylor, T.Y.R. Lee, “Thermal Expansion - Nonmetallic Solids", Plenum, New York, 1977.

13. H Kinoshita, S Otani, S Kamiyama, H Amano, I Akasaki, J Suda, H Matsunami, Jpn. J. Appl. Phys., 40, L1280 (2001). 